\title{
Holonic and multi-agent technologies for service and computing oriented manufacturing
}

\author{
André Thomas $^{1}$. Theodor Borangiu ${ }^{2}$. Damien Trentesaux ${ }^{3}$
}

Received: 21 December 2015 / Accepted: 22 December 2015 / Published online: 31 December 2015

(C) Springer Science+Business Media New York 2015

This special feature aims at shedding light on new emerging holonic and multi-agent systems operating in a service- and computing oriented manufacturing environment, using the latest ICT technologies such as service-orientation, mobile agents, Web- and Cloud services, virtualization, big data and analytics to name a few.

Industrials are seeking for models and solutions that are not only able to provide efficient overall production performance, but also to face reactively a growing set of unpredicted events. The demand for large scale industrial systems running in complex and even chaotic environments requires the consideration of new paradigms and technologies that provide flexibility, robustness, agility and responsiveness. Holonic systems are, actually by definition, targeting challenges that include coping with the heterogeneous nature of manufacturing systems and their on-line interactive nature in combination with competitive pressures. Multi-agent systems is a suitable implementing approach to address these challenge by offering an alternative way to design control systems, based on the decentralization of control functions over distributed autonomous and cooperative entities.

André Thomas

andre.thomas@univ-lorraine.fr

Theodor Borangiu

theodor.borangiu@cimr.pub.ro

Damien Trentesaux

damien.trentesaux@univ-valenciennes.fr

1 Université de Lorraine, 34 Cours Léopold, 54000 Nancy, France

2 Université Politehnica de Bucarest, Splaiul Independenţei 313, 060042 Bucharest, Romania

3 Université de Valenciennes, Voirie Communale Université Val Mont Houy, 59300 Famars, France
Moreover, virtualization of manufacturing execution system workloads offers a set of design and operational advantages to enterprises, the most visible being improved resource utilization and flexibility of the overall solution. At the manufacturing execution system level, cloud computing adoption refers mainly to virtualization of MES workloads. While MES implementations are different and usually depend directly on the actual physical shop floor layout, the general MES functions are aligned with the set of functions defined by ISA-95.03 specification. To achieve high levels of productivity growth and agility to market changes, manufacturers will need to leverage Big Data sets to drive efficiency across the networked enterprise. There is need for a framework allowing the development of manufacturing cyber physical systems that include capabilities for complex event processing and Big Data analytics, which are expected to move the manufacturing domain closer to digital transformation and cloud services within the contextual enterprise.

On the other hand, service orientation is emerging at multiple organizational levels in enterprise business, and leverages technology in response to the growing need for greater business integration, flexibility and agility of manufacturing enterprises. Close related to IT infrastructures of Web Services, the Service Oriented Enterprise Architecture represents a technical architecture, a business modelling concept, an integration source and a new way of viewing units of control within the enterprise. Business and process information systems integration and interoperability are feasible by considering the customized product as "active controller" of the enterprise resources-thus providing consistency between material and informational flows. The areas of Service Oriented Computing and Multi-agent Systems are getting closer, both trying to deal with the same kind of environments formed by loose-coupled, flexible, persistent and distributed 
tasks. An example is the new approach of Service Oriented Multi-agent Systems (SoMAS).

Following the 4th edition of the workshop SOHOMA 2014 in Nancy, this special feature contents four papers illustrating Holonic and Multi-Agent technologies for service and computing oriented manufacturing.

One important research activity in the field focuses on holonic manufacturing control implemented with multiagent systems that couple predictive/proactive and reactive mechanisms into agents/holons. All mechanisms leading to establish predictive plans and to react to unpredicted events occurring on the shop floor will have a distributed approach. Meanwhile, not enough attention is paid to the optimization of this coupling. The aim of the first paper entitled "Coupling predictive scheduling and reactive control in manufacturing hybrid control architectures: state of the art and future challenges", written by Olivier Cardin, Damien Trentesaux, André Thomas, Pierre Castagna, Thierry Berger, and Hind Bril El Haouzi, proposes a literature review useful to depict the main research challenges that are to be addressed before expecting a large industrial dissemination. Relying on an extensive review of the state of the art, three main challenges are highlighted: the estimation of the future performances of the system in reactive mode, the design of efficient switching strategies between predictive and reactive modes and the design of efficient synchronization mechanisms to switch back to predictive mode.

The second paper of this special feature, entitled "Resource scheduling based on energy consumption for sustainable manufacturing", has been written by Silviu Raileanu, Florin Anton, Alexandru Iatan, Theodor Borangiu, Silvia Anton, and Octavian Morariu. It proposes an agent-based approach for measuring in real time energy consumption of resources in job-shop manufacturing processes. Data from industrial robots is collected, analysed and assigned to operation types, and then integrated in an optimization engine in order to estimate how alternating between makespan and energy consumption as objective function affects the performances of the whole system. This study focuses on the optimization of energy consumption in manufacturing processes through operation scheduling on available resources. The decision making algorithm relies on a decentralized system collecting data about resources's status and quality of services they provide, implementing thus an intelligent manufacturing control system; the optimization problem is implemented using IBM ILOG OPL.

Offshore wind farms are a growing source of energy, which aims to ensure clean energy with a low environmental impact. In this context, the third paper entitled "Impact of spare parts remanufacturing on the operation and mainte- nance performance of offshore wind turbines: A multi-agent approach", written by Mohammed Dahane, M'hammed Sahnoun, Belgacem Bettayeb, David Baudry, Hamza Boudhar, investigates opportunities of the turbine gearbox end of lifecycle to improve the operation and maintenance strategies. It highlights how to determine the impact of spare part policy based on the remanufacturing of gearboxes recovered after each replacement. In this context, the remanufacturing implementation allows the extension of the gearbox life-cycle and involves a better $\mathrm{t}$ organization and coordination between maintenance, monitoring, operation and spare part supply chain to determine the best way to use each gearbox of each wind turbine. Finally, this paper presents a multi-agent based approach to analyze the impact of the spare parts remanufacturing strategy on the performance of an offshore wind farm in term of total cost and carbon footprint.

Supply chain network design and programming is a current issue that many practitioners have focused on and provided novel contributions. The fourth and last paper of this special feature, entitled "Evolutionary Fuzzy Intelligent System for Multi-Objective Supply Chain Network Designs: An Agent-based Optimization State of the Art" written by Mosahar Tarimoradi, M. H. Fazel Zarandi, Hosain Zaman, I.B. Turksan, presents a state of the art of agent-based optimization techniques. It puts forward a class of fuzzy multi-agent systems capable to transpose in practice decision makers' strategies with adaptation to the value chain environment. The main capabilities of this system reside in the facts that it interactively fulfills the decision makers' recommendations based on its robustness in optimization, self-tuning and learning facilities, adaptation to and awareness of the changes in environment.

Holonic Manufacturing Systems (HMS) and ServiceOriented Architectures (SOA) are currently two of the most studied and referenced solutions for the next generation of manufacturing systems; both of these solutions provide the necessary guidelines to create open, flexible and agile control environments for the smart, digital and networked factory.

The papers of this special feature have highlighted that already several industrial and scientific applications in the domain of holonic manufacturing have been implemented, have proven their reliability and capacity to rend manufacturing processes sustainable. Nevertheless a lot of challenges (scientific as well as applicative) still exist and especially concerning the joint use of predictive and reactive distributed control systems. The future of these research works will have to determine how, when and for what the control should switch between traditional ERP systems and new control systems with distributed intelligence. 